\title{
Sexual Attitudes among College Students: An Approach in Mexico
}

\author{
Aurora Montañez-Frausto ${ }^{1}$, Alicia Álvarez Aguirre ${ }^{2}$, Georgina Olvera-Villanueva ${ }^{3}$, \\ Ma. Teresa Hernández Ramos ${ }^{1}$, Nereyda Hernández Nava ${ }^{4}$, Ma. del Rocio Rocha-Rdz ${ }^{5}$ \\ ${ }^{1}$ Department of Nursing and Midwifery, Health Sciences Division, Campus León, University of Guanajuato, \\ Guanajuato, Mexico \\ ${ }^{2}$ Nursing Department, Campus Health Sciences and Engineering, University of Guanajuato, Guanajuato, \\ Mexico \\ ${ }^{3}$ School of Nursing, Autonomous University of Queretaro, Santiago de Querétaro, Mexico \\ ${ }^{4}$ Department of Nursing, Academic Coordination Altiplano Region, Autonomous University of San Luis Potosi, \\ San Luis Potosi, Mexico \\ ${ }^{5}$ Department of Nursing, Multidisciplinary Academic Unit Central Zone, Autonomous University of San Luis \\ Potosi, San Luis Potosi, Mexico \\ Email: rocio@uaslp.mx, rociorocha8@hotmail.com
}

Received 21 May 2015; accepted 18 July 2015; published 21 July 2015

Copyright (C) 2015 by authors and Scientific Research Publishing Inc.

This work is licensed under the Creative Commons Attribution International License (CC BY). http://creativecommons.org/licenses/by/4.0/

\begin{abstract}
Objective: To develop a predictive model for HIV/AIDS in the sexual behavior of university students from a public university in the state of Guanajuato. Methodology: Correlational study involved 294 university students from 18 to 19 years of age by stratified random sampling. The instruments used were: attitudes, subjective norms, self-efficacy and intentions for HIV/AIDS and sexual behavior. Descriptive statistics, normality test of Kolmogorov-Smirnov test, Spearman correlation and logistic regression was used. Results: In describing sexual behavior of young students at the beginning of sexual life is observed that $61.9 \%$ of them have had sexual life, with higher percentage in men. Of the 182 university students who reported first sexual intercourse used a contraceptive method 138 and 135 specifically used a condom the first time they had sex. The variables that have the ability to predict the sex drive at the start of sexual life, are the attitudes and perceived behavioral control $\left(X^{2}=39,638, \mathrm{df}=4, \mathrm{p}<0.000\right)$, reporting a $12.6 \%$ of explained variance. Conclusions: In general, university students today are perceived as vulnerable to sexual behavior and have safe sex, which leads us to think that protect not get HIV/AIDS and unwanted pregnancies.
\end{abstract}

\section{Keywords}

HIV/AIDS, Youth, Sexual Behavior, Attitudes, Intentions, Subjective Norms 


\section{Introduction}

Infections by the Human Immunodeficiency Virus (HIV) that causes Acquired Immunodeficiency Syndrome (AIDS) are the most widespread pandemic of the century and today. It is estimated that there are now 35.3 million people living with HIV. Global estimates confirm that in 2012, the number of new HIV infections is 2.3 million, a downward trend of 33\% of the 3.4 million it had in 2001, but still not enough. At the same time, the number of AIDS deaths also declined to 1.6 million in 2012, down from 2.3 million in 2005 [1].

With respect to age and its relationship with HIV, worldwide, about half of infections occur between 15 and 24 years, with 50\% of cases in women [1] [2]. In Latin America, it is estimated that the epidemic of the number of people aged 15 or older who acquired HIV infection in the world began in the early 1980s. In late 2011, it was 2.2 million, of which 740,000 are young between 15 and 24 years and about 1.5 million occurred in sub-Saharan Africa. The HIV epidemic continues stabilization trends and about 100,000 people become infected in Latin America, infecting primarily the gay people, injecting drug users (IDUs) and those engaged in sex work (PTS), especially working men sex (HTS) [3].

In Mexico, the first case of human immunodeficiency virus (HIV) was diagnosed in 1983 and in 2012 ranked third in the number of reported AIDS cases in the Latin America [4]. As of June 2013, the number of new AIDS cases is 2114 and amounted to 164,422 total reported cases, with a gender distribution of 134,946 men (82.1\%) and 29,476 women (17.9\%) with a male to female ratio of 4.6:1. Cases AIDS among young people 15 - 24 years of age; in 2013 they reported 19,314,000 cases, of which 14,734 (76.28\%) are men and 4580 (23.71\%) women with a male to female ratio of 3.2:1.5.

For Mexico, it has been reported that most of the cases have occurred in the group of 25 - 39 years old [5] [6]. From this we can conclude that the group most vulnerable to this disease are the young adults, because the time between contracting HIV and developing AIDS is about 5 to 10 years, you could say that most of these infections occur during adolescence.

One of the states of Mexico that has been most affected by this disease is Guanajuato due to the high rate of emigration at the stage of late adolescence. In this state, a total of 70 new AIDS cases were filed in the year of 2012, occupying the city of Irapuato second and third with more cases in the state since 2008 to date [7]. Until July 2013 AIDS cases, it is 3398 and the gender distribution is given as follows: 2845 men (83.72\%) and 553 females (16.28\%) with a male to female ratio of 4.5:1.5.

State Coordination of HIV/AIDS Guanajuato, 2012 Program reported that the average age of HIV-related deaths is 25 to 44 years old and according to the highest level of education the highest number of cases were located in those who only had studied primary (18.63\%) and marital status that prevailed at diagnosis was single (42.47\%). Among the most mentioned risk factors they were heterosexual (29.41\%) relationships, men who have sex with men $(15.01 \%)$ as well as (12.97\%) of bisexual relationships.

Therefore, it is important to combat this disease, study the university students as it is a population that is growing in number, which begins to be independent and economically active, which makes them more vulnerable.

Young people are particularly vulnerable to HIV infection and other sexually transmitted infections (STIs), as they are in a transition period in which they are no longer children but have not reached adulthood, leading changes this period are confused because they may not know what is the best decision to be taken in each of the activities of daily life. Social, emotional and psychological development is incomplete, so they tend to experience forms of dangerous behavior that can lead to serious consequences [8]-[10].

Many of these behaviors and consequences include unprotected sex (without a condom), sex with multiple partners and intravenous drug use and STIs and unwanted pregnancy [11].

In youth, we have a pattern of unpredictable behavior, lack the discernment that comes with age, usually they cannot appreciate the adverse consequences of their actions [12]. Also, because of the long incubation period has not risky behavior immediately apparent consequences so many young people engage in sexual risk behaviors such as unprotected sex, more than one sexual partner, sex under the influence of alcohol and drugs [13] [14]. These findings manifest that young people are not considered vulnerable to HIV/AIDS.

It has been proved that the conduct of the young can be influenced by beliefs due to his mental and subjective representations of reality and positively or negatively affect their actions. Normative beliefs have been relevant when it has studied the prevention of HIV/AIDS among adolescents, more particularly subjective norms that greatly influence the behavior of the young. At the same time, social norms and expectations, along with the 
view of colleagues, cultural and religious characteristics of familialism powerfully influence the behavior of young people, usually in a way that contributes to increased health risks [15].

According to the above, the present study is to develop a predictive model for HIV/AIDS in the sexual behavior of young people from a public university in Mexico purpose.

The following research question: What is the effect of attitudes, subjective norms, perceived control of behavior, intentions for HIV/AIDS in the sexual behavior of young college arises?

\section{Methods}

A descriptive study, performed correlational and cross. The study population was formed by 1166 university students between 18 and 19 years of the Division of Life Sciences, Campus Irapuato Guanajuato state Salamanca and correspond to the first 3 semesters of different careers at level degree: agricultural mechanics, environmental engineering, food engineering, agribusiness, veterinary medicine, renewable energy, engineering, agronomy and nursing. Stratified random sampling two strata by gender and half, proportional to the size of each stratum allocation was made (see Table 1). The total sample size was calculated using the formula for finite populations with a confidence level of 95\%, obtaining a sample of 294 university students.

Sexual Attitudes Scale 36 is designed to measure the conservative and liberal attitude toward sexual behavior was used. This instrument has 25 reactive written by way of statements of which two of them are worded negatively so it will be necessary to recode. An example statement would be: "There is no excuse for sex outside of marriage.” Response options for statements are: 0 = strongly disagree; 1 = Disagree; 2 = Neither agree nor disagree; 3 = Agree and $4=$ strongly agree. So the scores range from 0 - 100, where higher score indicates more conservative attitude about sex and the lowest score indicates a more liberal attitude. The estimated time to complete the instrument was 5 - 7 minutes. The reliability of the instrument is adequate, presenting a coefficient of internal consistency with a Cronbach's alpha of 0.94 , also the instrument has been validated through a point biserial correlation between the conservative and the liberal group $\mathrm{r}$ group $=0.73$. This instrument has been used in older Mexican adolescents of 18 - 24 years (Ramos, Rico and Benavides (2011). For the analysis of the information was generated a database through the Statistical Package for the Social Sciences (SPSS) 34 versión 19 for Windows 0.39 proceeded to check the internal consistency of each of the instruments through the reliability coefficient Cronbach Alpha.

\section{Results}

Following are the results in order to develop a predictive model (attitudes, subjective norms, perceived behavioral control, and intention) for HIV/AIDS in the sexual behavior of university students from a public university in Mexico are presented.

Sociodemographic characteristics of the participants. Table 1 shows the results of the sociodemographic

Table 1. Sociodemographic characteristics of university students.

\begin{tabular}{ccc}
\hline Variable & $\boldsymbol{f}$ & $\%$ \\
\hline Genre & 147 & 50 \\
Female & 147 & 50 \\
Male & & \\
Semester & 69 & 23.5 \\
First & 159 & 54.1 \\
Second & 66 & 22.4 \\
Third & & \\
Marital status & 289 & 98.3 \\
Single & 2 & 10.7 \\
Married & 3 & 1 \\
Separate & & \\
Working & 65 & 22.1 \\
Yes & 229 & 77.9 \\
Not & & $\mathrm{n}=294$ \\
Source: Direct & & \\
\hline
\end{tabular}


characteristics of the 294 university students who formed the study sample is. Their age ranged between 18 and 19 years with a mean of $18.55(\mathrm{SD}=0.498) .50 \%(\mathrm{f}=147)$ corresponded to females and $50 \%(\mathrm{f}=147)$ were males. Regarding grade, $23.5 \%(\mathrm{f}=69)$ enrolled in the first half, $54.1 \%(\mathrm{f}=159)$ the second half and $22.4 \%(\mathrm{f}=$ 66) the third semester. The races that young people are currently enrolled: agricultural mechanical engineering $2.4 \%(f=7)$, Environmental Engineering $10.5 \%(f=31)$, food engineering $20.1 \%(f=59)$, agribusiness $26 \%$ ( $f$ $=8.8$ ) veterinary medicine $10.5 \%(f=31)$, renewable energy $5.4 \%(f=16)$, engineering in agriculture $26.5 \%(f$ $=78)$ and nursing $15.6 \%(f=46)$. Further notes that $98.3 \%(f=289)$ of university students stated being single and $77.9 \%(f=229)$ was reported not to work.

Description of the sexual behavior of university students. Sexual behavior was assessed through a series of questions about the onset of sexual life and sexual behavior ever in life. The start of the sexual life of young students was measured with the question, Have you had sex at least once in life? shows the results on the frequency of onset of sexual life of young university, where it is observed that $61.9 \%$ of them have had sexual life, with the highest percentage (67.3\%) in young male, though this difference it is not significant $\left(\mathrm{X}^{2}=3.69\right.$; $\mathrm{p}=$ 0.055).

They were asked about whether his choice was to protect the condom or other contraception (IUD, diaphragm, pills, injections and/or foam) in the first time they had sex. Of the 182 university students who reported onset of sexual life, $46.9 \%$ ( $f=138$ ) used a contraceptive method and specifically $45.9 \%$ ( $f=135$ ) used a condom the first time they had sex. According to the data presented above about sexual conduct it can be summed up more than half of university students have already started their sexual life, especially in the male, but the difference is not significant. Most have chosen as a method of protection condom during their first sexual relation to care. After vaginal sex, oral sex is mostly practiced. With these results it achieves the objective one is to describe the sexual behavior of university students.

The variable of attitudes shows a median of 48 ; the score indicates that university students have a more liberal attitude about sex. Subjective norms also show a median of 59.72; which it means that in general young people tend to perceive that they have the approval of others like her father, her mother, her partner, friends and religion to perform sexual behavior. With respect to the variable control of the perceived behavior shows a median 90.34; these young people perceive control over to say no to sex, to ask the partner about previous sexual relations, to acquire and properly use a condom and maintain virginity until marriage, being faithful to one partner and talk about sex with parents. Regarding intentions variable sample medium 50, which means that young people have greater intention to make the protective behavior for HIV/AIDS (see Table 2).

Importantly, the data show a large standard deviation reflecting a large variability in responses; This becomes evident if we see that the minimum value for attitudes was of 0.00 ; meaning that no young university has a conservative approach with respect to its maximum value of 100 means that there are young people with a liberal attitude. Subjective norms for the minimum value was 0 ; meaning that there were young people who do not receive approval from others about their sexual behavior and in relation to the maximum value 93 means that there were young people who perceive greater approval of others regarding their sexual behavior.

To understand the relationship of attitudes, subjective norms, perceived behavioral control, intention to HIV/AIDS in the sexual behavior of university students, the correlation of Spearman's rho was used. Before analyzing the index for variables attitudes, subjective norms, perceived behavioral control and intentions it was built.

The results in Table 3 showed that attitudes to HIV/AIDS related negatively with the following sexual behaviors: sex at least once in life (rho $=-0.276, \mathrm{p}<0.000$ ), forced (a) the first time they had sex (rho $=-266$; $\mathrm{p}<$

Table 2. Descriptive statistics and results Kolmogorov-Smirnov test for instrument attitudes, subjective norms, perceived behavioral control and intentions for HIV/AIDS.

\begin{tabular}{lccccccc}
\hline & $\bar{X}$ & $\boldsymbol{D E}$ & $\boldsymbol{M d n}$ & Valor mínimo & Valor mínimo & KS & $\mathbf{p}$ \\
\hline Guidelines for HIV/AIDS & 48.53 & 10.53 & 48 & 0 & 100 & 1.526 & 0.019 \\
Subjective standards for HIV/AIDS & 58.33 & 15.17 & 59.72 & 0 & 93 & 1.148 & 0.143 \\
Perceived behavioral control HIV/AIDS & 87.68 & 24.76 & 90.34 & 25 & 133 & $\mathbf{1 . 2 4 3}$ & $\mathbf{0 . 0 9 1}$ \\
Intentions for HIV/AIDS & 49.19 & 20.09 & 50 & 6 & 100 & $\mathbf{1 . 5 8 6}$ & $\mathbf{0 . 0 1 3}$ \\
Source: Direct & & & & & & $\mathrm{n}=294$ \\
\hline
\end{tabular}


Table 3. Relationship between sexual behavior, attitudes, subjective norms, behavior management and intentions perceived HIV/AIDS.

\begin{tabular}{|c|c|c|c|c|c|c|c|c|c|}
\hline \multirow{3}{*}{ Sexual Behavior } & \multicolumn{7}{|c|}{ Subjectives } & & \\
\hline & \multirow[t]{2}{*}{$\mathbf{n}$} & \multicolumn{2}{|c|}{ Attitudes } & \multicolumn{2}{|c|}{ Standards } & \multicolumn{2}{|c|}{ Perceived behavioral control } & \multicolumn{2}{|c|}{ Intentions } \\
\hline & & $r_{h o}$ & $\mathbf{p}$ & $r_{h o}$ & $\mathbf{p}$ & $r_{h o}$ & $\mathbf{p}$ & $r_{h o}$ & $\mathbf{p}$ \\
\hline Have you had sex at least once in life? & 294 & -0.276 & 0 & 0.164 & 0.005 & -0.254 & 0 & -0.15 & 0.01 \\
\hline $\begin{array}{l}\text { The first time you had sex, your partner } \\
\text { forced you against your will? }\end{array}$ & 294 & -0.266 & 0 & 0.134 & 0.021 & -0.253 & 0 & -0.123 & 0.035 \\
\hline $\begin{array}{l}\text { The first time you had sex, } \\
\text { did you use birth control? }\end{array}$ & 182 & 0.131 & 0.077 & 0.052 & 0.482 & -0.054 & 0.472 & -0.062 & 0.407 \\
\hline $\begin{array}{l}\text { The first time you had sex, } \\
\text { did you use a condom? }\end{array}$ & 182 & 0.116 & 0.118 & 0.041 & 0.586 & 0.008 & 0.918 & -0.122 & 0.1 \\
\hline $\begin{array}{l}\text { Have you ever in your life } \\
\text { you had anal sex? }\end{array}$ & 294 & -0.121 & 0.038 & 0.09 & 0.122 & -0.139 & 0.017 & 0.019 & 0.749 \\
\hline $\begin{array}{l}\text { Have you ever in your life you } \\
\text { were given oral sex to someone else? }\end{array}$ & 116 & -0.047 & 0.617 & -0.037 & 0.693 & -0.064 & 0.493 & 0.058 & 0.535 \\
\hline Source: Direct & & & & & & & & & $n=294$ \\
\hline
\end{tabular}

0.000 ) and had anal sex at some time in life (rho $=-121 ; \mathrm{p}<0.038$ ). Meaning that by not making these sexual behaviors are related to a conservative attitude.

Also subjective standards for HIV/AIDS is positively related to the following sexual behaviors: sex at least once in life (rho $=0.164, \mathrm{p}<0.005$ ), forced (a) the first time they had sex (rho $=0.134, \mathrm{p}<0.021$ ); which means that failure to perform certain sexual behaviors, increased perception of approval (as her father, her mother, her partner, friends and religion) to conduct sexual behavior.

With regard to the perceived behavior control HIV/AIDS is associated negatively with the following sexual behaviors: having sex once in life (Rho $=-254 ; \mathrm{p}<0.000$ ), being forced (to) the first time they had sex (rho $=$ -253 ; $\mathrm{p}<0.000$ ) and if you have had anal sex at some time in life (rho $=-139$; $\mathrm{p}<0.017$ ); which means that not carrying out these sexual behaviors, they have more control perceived to say NO to sex under different circumstances, so it is negatively related to the question of whether the partner had previous sexual relations and other risk behaviors such as drug use, acquire and properly use a condom and maintain virginity until marriage.

The intentions are related negatively with the following sexual behaviors: Sexual ever in life (Rho $=-150$; $\mathrm{p}$ $<0.010$ ) relations, forced (a) the first time they had sex (rho $=-123$; $\mathrm{p}<0.035$ ); which means that not carrying out these behaviors intention of making the protective behavior for HIV/AIDS.

\section{Discussion}

In this study, the empirical application of the theoretical concepts of attitudes, subjective norms, perceived behavioral control, intention and sexual behavior was feasible. These concepts were the basis to develop a predictive model in the sexual behavior of 294 young people from a public university in the state of Guanajuato.

The results of this study showed that more than half of university students have already started their sexual life, especially in the male, but the difference is not significant. Most have specifically chosen as a method of protection condom during their first sexual relation to care. After vaginal sex, oral sex is mostly practiced.

Gender was a variable that was not significantly associated with the onset of sexual intercourse. In describing sexual behavior of young students at the start of sexual life, it was observed that more than half of the participants had sexual life, with greater percentage in young men, but this difference was not significant. This finding differs from that reported by Yip et al., (2013) and Pham et al., (2013), who showed significant difference in the onset of sexual activity by gender.

Most use a contraceptive method, and more specifically half used a condom the first time they had sex. This result is similar to that reported by Pham, et al., (2013) who showed that more than half of the study participants reported condom use.

One in seven was forced the first time they had sex. This finding is above that reported by Yip et al., (2013) who in their results sampled young people were forced to have sex with your partner. 
With respect to the relationship of attitudes to HIV/AIDS and sexual behavior of sex ever in life relationships found between variables negatively related. This result differs from that reported by Yip et al., (2013) which shows that favorable attitudes were positively associated toward premarital sex.

Regarding attitudes and use of a condom the first time sex relate negatively. This result differs from that reported by Harris, A., Sutherland, M. \& Hutchinson, K. (2012), which shows that attitudes towards condom use are statistically significant.

With regard to the intentions no relationship with sex at some time in life and condom use was found. This finding differs from that reported by William, Gunesekaran and Sekar (2013) which shows that the intention of having sex before marriage is significant.

University students today are perceived as vulnerable to sexual behavior and have safe sex, which leads us to think that having sex with no protection for getting HIV/AIDS or unwanted pregnancies.

\section{References}

[1] Joint United Nations Program on HIV/AIDS (2012) HIV Infection Rates Decreasing in Several Countries but the Global Number of People Living with HIV Continue to Rise. Journal of Adolescent Research, 19, 677-697.

[2] World Health Organization (2010) HIV/AIDS and Adolescents. 12, 203. http://www.who.int/features/factfiles/hiv/facts/en/index.html

[3] ONUSIDA (2012) Together We Will End AIDS. In: Twwe, W.L., Ed., 243640th Edition, WHO Library Cataloguing-in-Publication, UNAIDS/JC2296E, 36-40.

[4] ONUAIDS (2001) The Global Strategy Framework on HIV/SIDA. ONUAIDS, Ginebra.

[5] CENSIDA (2013) Epidemiology of HIV/AIDS Surveillance in Mexico. National Registry of AIDS Cases, 11, 2-23.

[6] Pilcher, C., Eron, J., Vemazza, P., Battegay, M., Yerly, S., Vom, S., et al. (2001) Sexual Transmission during the Incubation Period of Primary HIV Infection. Journal of the American Medical Association, 3, 286-295.

[7] State Coordination of HIV/AIDS Program Guanajuato (2012) Registro Estatal de casos nuevos de 2008 a julio 2013.3 , 4-12.

[8] Ramos, V., Rico, R., Martínez, P. and Benavides, R. (2011) Subjective Standards for HIV/AIDS and Sexual Behavior in Adolescents. Enfermería Global, 10, 677-697.

[9] William, T., Gunesekaran, S. and Sekar, I. (2013) Perceived Risk Sexual Behavior among Rural Youth. Golden Research Thoughts, 2, 1-10.

[10] Harris, A., Sutherland, M. and Hutchinson, K. (2013) Parental Influences of Sexual Risk among Urban African American Adolescent Males. Journal of Nursing Scholarship, 45, 141-150. http://dx.doi.org/10.1111/jnu.12016

[11] Pulido, M., Carazo, V., Orta, G., Coronel, M. and Vera, F. (2011) Risky Sexual Behavior in Undergraduates of the Intercontinental University. Revista Intercontinental de Psicología y Educación, 1, 11-27.

[12] Pineda, J., Ramos, M., Frias, M. and Cantu, P. (2000) Survey on Adolescent Reproductive Health and Higher Education Students in Monterrey. Revista Salud Publica y Nutrición, 1.

[13] López, F. and Moral de la Rubia, J. (2001) Validation of a Scale of Self-Efficacy for AIDS Prevention in Adolescents. Salud Pública de México, 43, 421-432.

[14] Palacios, J., Bravo, M. and Andrade, P. (2007) Alcohol and Sexual Risk Behavior in Adolescents. International Psychology, 18, 1-13.

[15] Benavides, R.A. (2007) An Interaction Model of Parents’ and Adolescents’ Influences on Mexican Adolescents' Intentions for Contraception and Condom Use. Tesis de Doctorado no Publicada, Universidad de Austín, Austín. 